\title{
Differential Invariants of One Parametrical Group of Transformations
}

\author{
Abdishukurova Guzal, Narmanov Abdigappar*, Sharipov Xurshid \\ Faculty of Mathematics, National University of Uzbekistan, Tashkent, 100174, Tashkent, Uzbekistan
}

Received March 6, 2020; Revised April 24, 2020; Accepted May 3, 2020

Copyright $\odot 2020$ by authors, all rights reserved. Authors agree that this article remains permanently open access under the terms of the Creative Commons Attribution License 4.0 International License

\begin{abstract}
The concept of differential invariant, along with the concept of invariant differentiation, is the key in modern geometry [1]-[10]. In the Erlangen program [3] Felix Klein proposed a unified approach to the description of various geometries. According to this program, one of the main problems of geometry is to construct invariants of geometric objects with respect to the action of the group defining this geometry. This approach is largely based on the ideas of Sophus Lee, who introduced continuous geometry groups of transformations, now known as Lie groups, into geometry. In particular, when considering classification problems and equivalence problems in differential geometry, differential invariants with respect to the action of Lie groups should be considered. In this case, the equivalence problem of geometric objects is reduced to finding a complete system of scalar differential invariants.
\end{abstract}

The interpretation of the $k$ - order differential invariant as a function on the space of $k-$ jets of sections of the corresponding bundle made it possible to operate with them efficiently, and using invariant differentiation, new differential invariants can be obtained.

Differential invariants with respect to a certain Lie group generate differential equations for which this group is a symmetry group. This allows one to apply the well-known integration methods to such equations, and, in particular, the LiBianchi theorem [4].

Depending on the type of geometry, the orders of the first nontrivial differential invariants can be different. For example, in the space $R^{3}$ equipped with the Euclidean metric, the complete system of differential invariants of a curve is its curvature and torsion, which are second and third order invariants, respectively.

Note that scalar differential invariants are the only type of invariants whose components do not change when changing coordinates. For this reason, scalar differential invariants are effectively used in solving equivalence problems.

In this paper differential invariants of Lie group of one parametric transformations of the space of two independent and three dependent variables are studied. It is shown method of construction of invariant differential operator.Obtained results applied for finding differential invariants of surfaces.

Keywords Lie Group of Transformations, Differential Invariant, Vector Field, Invariant Differential Operator

\section{Introduction}

Let $G$ be a Lie group of transformations of Riemannian manifold $M$ of dimension $n$ with Riemannian metric $g$.

Definition 1. A function $F(x)$ is called an invariant of the group of transformations of $G$ if $F(x)=F(g x)$ for each element $g \in G$.

Example 1. Consider the group of motions of the Euclidean plane

$$
X^{t}:(x, y)=(x \cos t+y \sin t ;-x \sin t+y \cos t),
$$

where $t \in R$. The function $F(x, y)=x^{2}+y^{2}$ is an invariant under the rotation group, since

$$
x^{2}+y^{2}=(x \cos t+y \sin t)^{2}+(-x \sin t+y \cos t)^{2}
$$

for all $t \in R$.

For the group of rotations of the Euclidean plane, the infinitesimal generator is vector field

$$
X=-y \frac{\partial}{\partial y}+x \frac{\partial}{\partial y} .
$$

Let $G$ be the Lie group, the transformations of the Riemannian manifold $M$. If the group $G$ is an $k$-dimensional Lie group, then it has the same $k$ infinitesimal generators (vector fields) [6]. The following theorem is known [8]:

Theorem 1. The function $F(x)$ is an invariant of the group of transformations of $G$ if and only if $X(F)=0$ holds for each infinitesimal generator $X$ of the group $G$.

Definition 2. Let $M, B$ be smooth manifolds and $p \in M$. Let $f, g: M \rightarrow B$ be smooth mappings satisfying the condition $f(p)=g(p)=q$. 
1) $f$ has a first-order tangency with $g$ at the point $p$ if $(d f)_{p}=$ $(d g)_{p}$ as the map $T_{p} M \rightarrow T_{p} B$.

2) $f$ has a contact of $k$ th order with $g$ at the point $p$ if the map $(d f): T M \rightarrow T B$ has a contact of order $(k-1)$ with map $(d g)$ at each point $T_{p} M$. This fact can be written as follows: $f \sim_{k} g$ at the point $p$ ( $k$-positive number) [2].

Example 2. Let $f, g: R \rightarrow R, f(x)=x^{3}, g(x)=x^{4}+x^{3}$. Then $f \sim_{2} g$ at the point $0(k=2)$. In fact, $f(0)=g(0)=$ $0, f^{\prime}(0)=g^{\prime}(0)=0, f^{\prime \prime}(0)=g^{\prime \prime}(0)=0$.

We denote by $J^{k}(M, B)_{p, q}$ the sets of equivalence classes with respect to the " $\sim_{k}$ at the point $p$ " in the space of mappings $f: M \rightarrow B$ satisfying the condition $f(p)=q$. We put $J^{k}(M, B)=\bigcup_{(p, q) \in M \times B} J^{k}(M, B)_{p, q}$.

Definition 3.The set $J^{k}(M, B)$ is called the space of $k$-jets.

The action of the group $G$ on $M$ gives rise to some action of the group on $J^{k}(M, B)$. This action is called the $k$ -prolongation of the group $G$ on $J^{k}(M, B)$.

We let $G^{(k)}$ denote the associated prolonged group action on the jet space $J^{k}(M, B)$. The infinitesimal generators of the $k$ -th prolongation of the group $G$ to $J^{k}(M, B)$ are $k$ - prolongations of infinitesimal generators of the group $G$ [9].

For example, the first and second prolongations of the vector field (3) are respectively vector fields

$$
X^{(1)}=-y \frac{\partial}{\partial x}+x \frac{\partial}{\partial y}+\left(1+y^{\prime 2}\right) \frac{\partial}{\partial y^{\prime}}
$$

and

$$
X^{(2)}=-y \frac{\partial}{\partial x}+x \frac{\partial}{\partial y}+\left(1+y^{\prime 2}\right) \frac{\partial}{\partial y^{\prime}}+3 y^{\prime} y^{\prime \prime} \frac{\partial}{\partial y^{\prime \prime}}
$$

Definition 4. The function $I \in C^{\infty}\left(J^{k}(M, B)\right)$ is called a differential invariant of order $k$ of the group $G$ if it is preserved under the action of the $k$-th prolongation $G$ on $J^{k}(M, B)$, that is, $g(I)=I$ for any transformation $g \in G^{(k)}$.

Differential invariants of Lie group of transformations are studied in the papers [1], [6]- [10].

The following theorem is known [8]:

Theorem 2. The function $I$ is an invariant of order $k$ of the transformation group $G$ if and only if it is the first integral of the infinitesimal generator of the group $G^{(k)}$.

\section{Main Part}

Let $G$ be a Lie group of transformations of the space of two independent $u, v$ and three dependent $x_{1}, x_{2}, x_{3}$ variables , and following vector field

$$
X=\xi_{1}(u, v, x) \frac{\partial}{\partial u}+\xi_{2}(u, v, x) \frac{\partial}{\partial v}+\sum_{i=1}^{3} \eta_{i}(u, v, x) \frac{\partial}{\partial x_{i}}
$$

is infinitesimal generator of the group $G$.

It is known that any Lie group is similar to the group of translations. This property of the groups is remarkable and its use permits simplification of finding of differential invariants of the group ([10],page 28).

In order to use this possibility we produce the replacement of variables.
Let us consider functions $F_{1}(u, x)$ and $F_{2}(v, x)$ which are solutions of following equation

$$
X(F)=1 .
$$

Let $I_{1}(u, v, x), I_{2}(u, v, x)$ and $I_{2}(u, v, x)$ be are functionally independent invariant functions of the group $G$, i.e. they are satisfy following equations

$$
X\left(I_{i}\right)=0, i=1,2,3 .
$$

We will replace the variables in the space of $\left(u, v, x_{1}, x_{3}, x_{3}\right)$ by putting

$$
\begin{gathered}
s=F_{1}(u, x), t=F_{2}(v, x), \\
y_{i}=I_{i}(u, v, x),
\end{gathered}
$$

where $i=1,2,3$. Using easy deductions, we can verify that in variables $\left(s, t, y_{1}, y_{2}, y_{3}\right)$ the vector field (6)has the following form

$$
X=\frac{\partial}{\partial s}+\frac{\partial}{\partial t} .
$$

This form of the vector field $X$ shows that the group $G$ is similar to the group of translations. Moreover in the coordinates $\left(s, t, y_{1}, y_{2}, y_{3}\right)$ for any $k \in N$ for $k-t h$ prolongation $X^{(k)}$ of the vector field (11) it holds equality $X^{(k)}=X$ [8].

Let us recall differentiation operator $D$ is called invariant differentiation operator with respect group $G$ if it holds $D X(F)=X D(F)$ for any smooth function $F[6]$.

It follows from the form of the vector field (11) invariant differentiation operators for the group $G$ are following operators of total derivatives: $D=D_{s}+D_{t}$.

If we put

$$
p_{i, k}=\frac{\partial^{k} y_{i}}{\partial s^{k}}, q_{i, k}=\frac{\partial^{k} y_{i}}{\partial t^{k}}
$$

then we can write total derivatives in following forms:

$$
\begin{aligned}
& D_{s}=\frac{\partial}{\partial s}+\sum_{i=1}^{3} p_{i, 1} \frac{\partial}{\partial y_{i}}+\sum_{i=1}^{3} p_{i, 2} \frac{\partial}{\partial p_{i, 1}}+\ldots \\
& D_{t}=\frac{\partial}{\partial t}+\sum_{i=1}^{3} q_{i, 1} \frac{\partial}{\partial y_{i}}+\sum_{i=1}^{3} q_{i, 2} \frac{\partial}{\partial q_{i, 1}}+\ldots
\end{aligned}
$$

We have the following equalities

$$
\begin{aligned}
& D_{s}=\frac{1}{D_{u} F_{1}} D_{u}, \\
& D_{t}=\frac{1}{D_{v} F_{2}} D_{v},
\end{aligned}
$$

which will allow us to return to the old variables, where $D_{u}, D_{v}$ - also operators of total derivatives with respect $u, v$.

Let denote by $D^{k}(F)$ derivatives $D_{s}^{k}+D_{t}^{k}(F)$ of order $k$.

Thus we have the following theorem.

Theorem-3. Suppose $I_{1}, I_{2}, I_{3}$ are independent invariants of the group $G, F_{1}(u, x), F_{2}(v, x)$, are solutions of the equation $X(F)=1$. Then functions $I_{i}(u, v, x)$ and $D^{k}\left(I_{i}\right)$ are differential invariants of order $k$. 
Let us find differential invariants of surfaces. We consider a surface which is given by equations

$$
x=x(u, v), y=y(u, v), z=z(u, v)
$$

in $R^{3}$.

We can put

$$
x=u, y=v, z=z(u, v)
$$

and get space of two independent $x, y$ and one dependent $z=z(x, y)$ variables. Let consider the group of conformal transformations of the Euclidean space $R^{3}(x, y, z)$

$$
X^{t}: R^{3} \rightarrow R^{3}
$$

where

$$
X^{t}(x, y, z)=\left(x e^{t}, y \exp (t), z \exp (t)\right),
$$

where $t \in R$, with infinitesimal generator

$$
X=x \frac{\partial}{\partial x}+y \frac{\partial}{\partial y}+z \frac{\partial}{\partial z} .
$$

The function

$$
I(x, y, z)=\frac{x^{2}+y^{2}}{z^{2}}
$$

is an invariant under the group (18).

From the equation

$$
X(F)=1
$$

we obtain

$$
\begin{aligned}
& F_{1}(x, z)=\frac{1}{2} \ln (x z) . \\
& F_{2}(y, z)=\frac{1}{2} \ln (x z) .
\end{aligned}
$$

We will replace the variables in the space of $(x, y, z)$ by putting

$$
s=F_{1}(x, z), t=F_{2}(y, z), w=I(x, y, z) .
$$

Now we can use formulas (14)and (15) to find differential invariants of the surface (16). By produce derived necessary calculations, we find the following first-order differential invariants

$$
I_{1}^{(1)}=\frac{x z_{x}^{\prime}}{z+x z_{x}^{\prime}}, I_{2}^{(1)}=\frac{y z_{x}^{\prime}}{z+y z_{x}^{\prime}} .
$$

By invariant differentiating the differential invariants of the first order, we obtain the second order differential invariants e

$$
\begin{aligned}
& I_{1}^{(2)}=2 x y z \frac{z z_{x y}^{\prime}-z_{x} z_{y}}{\left(z+x z_{x}^{\prime}\right)^{2}\left(z+y z_{y}\right)}, \\
& I_{2}^{(2)}=2 x y z \frac{z z_{x y}^{\prime}-z_{x} z_{y}}{\left(z+y z_{y}^{\prime}\right)^{2}\left(z+x z_{x}\right)}, \\
& I_{3}^{(2)}=2 x z \frac{z\left(z_{x}+x z_{x x}\right)-x z_{x}^{2}}{\left(z+x z_{y}^{\prime}\right)^{3}},
\end{aligned}
$$

Taking into account that the obtained differential invariants are functions of other invariants, we can find more simple differential second-order invariants:

$$
I_{4}^{(2)}=\frac{x^{2} z_{x x}}{z+x z_{x}},
$$

$$
\begin{aligned}
& I_{5}^{(2)}=\frac{x y z_{x y}}{z+x z_{x}}, \\
& I_{6}^{(2)}=\frac{x y z_{x y}}{z+y z_{y}}, \\
& I_{7}^{(2)}=\frac{y^{2} z_{y y}}{z+y z_{y}} .
\end{aligned}
$$

Let $G$ be a group of motions of the Euclidean space $R^{3}(x, y, z)$, where two independent $x, y$ and one dependent $z$ variables

$$
X^{t}: R^{3} \rightarrow R^{3}, t \in R,
$$

where

$$
X^{t}(x, y, z)=(x \cos t+z \sin t, y+t,-x \sin t+z \cos t)
$$

with infinitesimal generator

$$
X=-z \frac{\partial}{\partial y}+\frac{\partial}{\partial y}+x \frac{\partial}{\partial z} .
$$

The function $I(x, z)=\sqrt{x^{2}+z^{2}}$ is an invariant under the actionof the group (20).

From the equation

$$
X(F)=1
$$

we obtain

$$
F_{1}(x, z)=\arcsin \frac{z}{\left[x^{2}+z^{2}\right]^{\frac{1}{2}}} .
$$

$$
F_{2}(y, z)=y \text {. }
$$

We will replace the variables in the space of $(x, y, z)$ by putting

$$
s=F_{1}(x, z), t=F_{2}(y, z), w=I(x, z) .
$$

Using easy deductions, we can verify that in variables $(s, t, w)$ the vector field (21) has the following form

$$
X=\frac{\partial}{\partial s}+\frac{\partial}{\partial t} .
$$

This form of the vector field $X$ shows that the group $G$ is similar to the group of translations.

Now we can use formulas (14)and (15) to find differential invariants of the surface (16). By produce derived necessary calculations, we find the following first-order differential invariants

$$
I_{1}^{(1)}=\frac{z z_{x}^{\prime}+x}{z-x z_{x}^{\prime}}, I_{2}^{(1)}=\frac{z z_{y}^{\prime}+y}{y z_{x}^{\prime}-z} .
$$

By invariant differentiating the differential invariants of the first order, we obtain the second order differential invariants

$$
\begin{gathered}
I_{1}^{(2)}=\frac{1+z_{x}^{2}}{x z_{x}^{\prime}-z}+z_{x x} \frac{x^{2}+z^{2}}{\left(x z_{x}-z\right)^{2}}, \\
I_{2}^{(2)}=\frac{1+z_{y}^{2}}{y z_{y}^{\prime}-z}+z_{y y} \frac{x^{2}+z^{2}}{\left(y z_{y}-z\right)^{2}}, \\
I_{3}^{(2)}=x z_{y} \frac{1+z_{x}^{2}}{\left(x z_{x}^{\prime}-z\right)^{2}}+z_{x y} \frac{x^{2}+z^{2}}{\left(x z_{x}^{\prime}-z\right)^{2}}, \\
I_{4}^{(2)}=\frac{z_{y}^{2}+z z_{y y}}{\left(x^{2}+z^{2}\right)^{\frac{1}{2}}}-\frac{z^{2} z_{y}^{2}}{\left(x^{2}+z^{2}\right)^{\frac{3}{3}}},
\end{gathered}
$$


Now we have following second order differential invariant which is functions of obtained second order differential invariants

$$
K^{(2)}=\frac{z_{x x} z_{y y}-z_{x y}^{2}}{\left(1+z_{x}^{2}+z_{y}^{2}\right)^{2}} .
$$

It is known second order differential invariant $K^{(2)}$ is Gauss curvature of the surface which is grafics of the function $z=$ $z(x, y)$.

\section{The case of a single independent vari- able}

Let us consider the case there is a single independent variable $x$ and single dependent variable $y$.

This case is considered in the paper [11]. In this case one consider function $F(y, x)$ which is solution of following equation

$$
X(F)=1 .
$$

Let $I(x, y)$ be a invariant function of the group $G$, i.e. it satisfy following equation

$$
X(I)=0 .
$$

We will replace the variables in the space of $(x, y)$ by putting

$$
\begin{aligned}
& s=F(x, y), \\
& z=I(x, y) .
\end{aligned}
$$

This case is shown in following example.

Example-3. Let us find the differential invariant of the rotation group of the plane (1). The finding of differential invariants reduces to finding the first integrals of the system of ordinary differential equations. By the theorem-2, the function $I$ is an invariant of the second order if and only if

$$
X(I)=X^{(1)}(I)=X^{(2)}(I)=0
$$

From these relations we obtain the following system of equations

$$
\frac{d x}{-y}=\frac{d y}{x}=\frac{d y^{\prime}}{1+y^{\prime 2}}=\frac{d y^{\prime \prime}}{3 y^{\prime} y^{\prime \prime}}
$$

From the equation $\frac{d x}{-y}=\frac{d y}{x}$, we get invariant function

$$
I=\sqrt{x^{2}+y^{2}} .
$$

From the equation

$$
X(F)=1
$$

we obtain

$$
F(x, y)=\arcsin \frac{x}{\sqrt{x^{2}+y^{2}}} .
$$

By using invariant differential operator we have first order differential invariant

$$
I_{1}=\frac{x y^{\prime}-y}{x+y y^{\prime}} .
$$

and second order differential invariant

$$
I_{2}=\frac{y^{\prime \prime}}{\left(1+y^{\prime 2}\right)^{3 \mid 2}} .
$$

This invariant is the curvature of the curve $y=y(x)$.

This case of single independent variable is also considered in papers [7]-[9]. As shown in this papers if $I\left(x, u^{(n)}\right)$ is any (non-constant) differential invariant, the corresponding invariant differential operator is $D=\frac{D_{x}}{D_{x} J}$ which maps $J$ to $\frac{d J}{d I}=D_{x} J / D_{x} I$. Therefore, starting from a pair of differential invariants (or, more generally, a single differential invariant and a contact- invariant horizontal one-form) we construct an infinite sequence of order differential invariants.

Let us apply this procedure for example above. We consider two invariants

$$
\begin{aligned}
& I=\sqrt{x^{2}+y^{2}}, \\
& I^{(1)}=\frac{x y^{\prime}-y}{x+y y^{\prime}} .
\end{aligned}
$$

of order 0 and first order respectively.In this case we have got

$$
\begin{gathered}
D_{x} I=\frac{x+y y^{\prime}}{\sqrt{x^{2}+y^{2}}}, \\
D_{x} I^{(1)}=\frac{\left.y^{\prime \prime}\left(x^{2}+y^{2}\right)+\left(1+y^{\prime 2}\right)\left(x y^{\prime}+y\right)\right)}{\left(x+y y^{\prime}\right)^{2}} .
\end{gathered}
$$

As result we have following second differential invariant

$$
I^{(2)}=\left(x^{2}+y^{2}\right) \frac{y^{\prime \prime}}{\left(x+y y^{\prime}\right)^{2}}+\frac{1+y^{\prime 2}}{\left(x+y y^{\prime}\right)^{2}} .
$$

We now first and second prolongations of the the infinitesimal generator for the group of rotations of the Euclidean plane (4),(5). So we can check that

$$
X^{(1)}\left(I^{(1)}\right)=0, X^{(2)}\left(I^{(2)}\right)=0 .
$$

From this equalities follows that functions $I^{(1)}$ and $I^{(2)}$ are differential invariants of first and second order respectively.

\section{Invariants of submersions}

In this section we discuss second order differential invariants of submersions.

Let us consider submersion

$$
H: R^{m} \rightarrow R^{1},
$$

i.e. differentiable function of maximal rank. According to the theorem on rank for every $c \in R^{1}$ full primage $H^{-1}(c)$ is a smooth manifold of dimension $n-1$. Thus connected components of level surfaces $L_{c}=\{x: H(x)=c\}$ generate foliation $F$ codimension one.

We want to find differential invariants of the group of one parametrical transformations which respects foliation $F$.

Let

$$
(x, y, z)=\left(x^{1}, x^{2} \ldots, x^{n}, y^{1}, y^{2} \ldots, y^{n}, z^{1}, z^{2} \ldots, z^{l}\right),
$$

cartesian coordinates in $R^{m}$ where $2 n+l=m$. If $F(x, y, z), H(x, y, z)$ are smooth functions, we define their Poisson bracket to be the function

$$
\{F, H\}=\sum_{i=1}^{n} \frac{\partial F}{\partial y^{i}} \frac{\partial H}{\partial x^{i}}-\sum_{i=1}^{n} \frac{\partial F}{\partial x^{i}} \frac{\partial H}{\partial y^{i}} .
$$


The space $R^{m}$ is a Poisson manifold with above defined Poisson bracket.

Definition -3. Let $M$ be a Poisson manifold and $H: M \rightarrow$ $R$ a smooth function. The Hamiltonian vector field associated with $\mathrm{H}$ is the unique smooth vector field $X_{H}$ on $M$ satisfying $X_{H}(F)=\{F, H\}=-\{H, F\}$ for every smooth function $F: M \rightarrow R$.

In this case of the canonical Poisson bracket on $R^{m}, m=$ $2 n+l$, the Hamiltonian vector field corresponding to $H(x, y, z)$ is clearly

$$
X_{H}=\sum_{i=1}^{n}\left\{\frac{\partial H}{\partial x^{i}} \frac{\partial}{\partial y^{i}}-\frac{\partial H}{\partial y^{i}} \frac{\partial}{\partial x^{i}}\right\}
$$

The corresponding flow is defined by the system of ordinary differential equations

$$
\frac{d x^{i}}{d t}=-\frac{\partial H}{d y^{i}}, \frac{d y^{i}}{d t}=\frac{\partial H}{d x^{i}}, \frac{d z^{l}}{d t}=0 .
$$

It is known that the function $H(x, y, z)$ first integral for the system (25). It follows from here the flow of the system (25) translates every leaf of the foliation $F$ onto it [5] .

Thus vector field $X_{H}$ generate the group $G$ of one parametrical transformations which respects foliation $F$. If we want to find differential invariants of the group $G$ one can use the function $H(x, y, z)$ as invariant.

Let us consider functions $F_{i}(x, y), i=1,2, \cdots, 2 n$, which are solutions of following equation

$$
X\left(F_{i}\right)=1 \text {. }
$$

The function $H(x, y, z)$ satisfy following equation

$$
X_{H}(H(x, y, z))=0 .
$$

We will replace the variables in the space of $(x, y, z)$ by putting

$$
s_{i}=F_{i}(x, y), z_{j}=z_{j},
$$

where $i=1,2, \cdots, 2 n, j=1,2, \cdots, l$. Using easy deductions, we can verify that in variables $(s, z)$ the vector field (24)has the following form

$$
X=\sum_{i=1}^{2 n} \frac{\partial}{\partial s_{i}}
$$

By applying differentila oprators (total derivatives) $D_{s_{i}}$ to the function $H(x, y, z)$ we can find differential invariants of the foliation, generated by submersion (22).

Now let us show this procedure on example. We consider the submersion

$$
H(x, y, z)=x^{2}+y^{2}-z .
$$

where $m=3, n=1, l=1$.

The Hamiltonian vector field corresponding to $H(x, y, z)$ is clearly is the vector field (3). From equations (26) we find

$$
F_{1}=\arctan \frac{y}{x}, F_{2}=\arcsin \frac{y}{\sqrt{x^{2}+y^{2}}}+z .
$$

We will replace the variables in the space of $(x, y, z)$ by putting

$$
s_{i}=F_{i}(x, y), z=z
$$

where $i=1,2$. In new system coordinate the vector field (3) has following form

$$
X=\frac{\partial}{\partial s_{1}}+\frac{\partial}{\partial s_{2}}
$$

By applying total derivative operator

$$
D_{s_{1}}+D_{s_{2}}
$$

to the function $H(x, y, z)$ we can find differential invariants of the foliation, generated by submersion (27).

Now as a result of small calculations we have following second order differential invariant

$$
K=\frac{4}{\left(1+4 x^{2}+4 y^{2}\right)^{2}},
$$

which is Gauss curvature of a level surface of the submersion (27) at the point $(x, y, z)$.

\section{Conclusion}

We have considered two problems in this paper. First problem is a problem on second order differential invariants of the one parametrical tranformations. Results on this problem obtained in this paper shows that how can be used the fact that any Lie group is similar to the group of translations. This property of Lie group is remarkable and its use permits simplification of finding of differential invariants of the group. But we have technical problems with finding functionally independent invariant functions of the group. Finding functionally independent invariant functions is a equivalent to the finding of first integrals of the system of ordinary differential equations. In general finding of first integrals of the system of ordinary differential equations is very complicated.

Second problem in this paper deals with geometry of submersions. The study of the geometry of submersions is closely related to the geometry of foliations, which is an important section of modern geometry. Submersion generates a foliation whose leaves are level surfaces of the submersion. Therefore, studying the geometry of submersions is important in the theory of foliations. We have pointed out how can be find geometrical characteristics of a submersion which are invariants of the Lie group action.

\section{Acknowledgements}

We are very grateful to experts for their appropriate and constructive suggestions to improve this template. 


\section{REFERENCES}

[1] Alekseevsky D.V., Vinogradov A.M., Lychagin V.V. Basic ideas and concepts of differential geometry. The results of science and technology. Modern problems of mathematics. Fundamental directions. - Moscow: VINITI. 1988-28.-298 p.

[2] Golubitskii M., Guiyemin V. Stability maps and their singularities. Moscow publishing house. Mir -1977

[3] Felix Klein. A comparative review of recent researches in geometry, trans. M. W. Haskell, Bull. New York Math. Soc. 2, (1892-1893), 215-249.

[4] Kushner A.G., Lychagin V.V., Rubtsov V.N. Con- tact geometry and nonlinear differential equations. Encyclopedia of Mathematics and Its Applications 101. Cambridge: Cambridge University Press. 2007. XXII,496 P.

[5] Narmanov Abdigappar, Parmonov Hamid, "On the Geometry of Hamiltonian Symmetries," Mathematics and Statistics, Vol. 8, No. 3, pp. 293 - 298, 2020. DOI: 10.13189/ms.2020.080307.
[6] Olver P. J. Differential invariants and invariant differen- tial equations, Lie Groups and their Appl. 1 (1994), 177-192.

[7] Olver P. J. Differential invariants of surfaces, Diff. Geom. Appl. 27 (2009), 230-239.

[8] Olver P. J. Applications of Lie Groups to Differential Equations.Springer, 1993.

[9] Olver P. J. Differential invariants, Acta Applicandae Math. 41 (1995), 271-284.

[10] Ovsiannikov L. Group Analysis of Differential Equa- tions. Academic Press, 1982

[11] Popovich R. E., Boyko B. N. Differensialnye inwarianty odnoparametricheskoy gruppy lokalnyh preobrazowaniy i integriruemye urawnenie Rikkati.Vestnik SamGu. 2001, no-4(18), 49-56 pages (in Russian). 